\title{
RESUMEN:
}

La forma de hacer publicidad ha cambiado. Los distintos públicos exigen a las empresas más cercanía y atención a las problemáticas que se presentan en la actualidad, por esto, la publicidad Below The Line, en adelante BTL, a través de formas no masivas de comunicación genera estrategias de mercadeo para determinados grupos de la población teniendo como objetivo dar a conocer o generar una intención de compra hacia un producto. Las cuales deben darse dentro de los parametros de la sostenibilidad. Que se entiende como el uso adecuado de los recursos y la correcta disposicion de los mismos para cumplir nuevos objetivos, es decir, entender el uso de los recursos como parte de una cadena que no termina con una sola utilización dentro de una única acción de mercadeo. Estas prácticas hacen parte de la Responsabilidad social empresarial, en adelante RSE, que es la contribución activa que las empresas hacen a la sociedad, economia y el medio ambiente donde desarrollan sus activdades. Por lo tanto, se identificará para el caso de la industria del Mercadeo BTL de la ciudad de Medellin si son aplicadas las prácticas mencionadas anteriormente, para asi generar estrategias aplicables al sub-sector objeto de este estudio.

Palabras Clave: Sostenibilidad, Responsabilidad social, Publicidad Below the line.

\section{LA IMPORTANCIA DEL CONOCIMIENTO SOBRE SOSTENIBILIDAD PARA LOS EMPRESARIOS DEL SECTOR DE LA PUBLICIDAD BELOW THE LINE EN LA CIUDAD DE MEDELLÍN COLOMBIA.}

\author{
Rafael Jaime Carmona López, / Edgar Camilo Cuadros Blanco / Juan Guillermo Pérez Rojas \\ Ricardo Espinoza Díaz
}

Fechas de recepción: 11 de diciembre de 2020

Fecha de aceptación: 23 de diciembre de 2020

DOI: https://doi.org/10.22370/riace.2020.9.1.2606

\footnotetext{
1 Doctor en Ciencias de la Administración, Magister En Ciencias de la Administración, de la Universidad de Santiago de Chile. M.B.A, de la Universidad Eafit. Administrador de Empresas. Profesor Investigador. Universidad Pontificia Bolivariana. Correo electrónico: Rafael.carmona@upb.edu.co 2 Negociador Internacional de la Universidad Pontificia Bolivariana, Medellín Col Director ALCUBO TRAVEL S A S is located in Medellín, Antioquia, Colombia.

Correo electrónico: edgar.cuaros@upb.edu.co

3 Magíster en Desarrollo. De la Universidad Pontificia Bolivariana. Filósofo, Rector del Instituto Tecnológico Metropolitano ITM. Correo electrónico: rector@itm.edu.co

4 Doctor (e) En Ciencias de la Administración de la Universidad de Santiago de Chile. Magister en Administración Universidad de Santiago de Chile, Jefe de Carrera de Ingeniería en Información y Control de Gestión, Universidad de Valparaíso. Correo electrónico: Ricardo.espinoza@uv.cl
} 


\title{
THE SUSTAINABILITY KNOWLEDGE IMPORTANT FOR ENTREPRENEURS OF BELOW THE LINE MARKETING SECTOR FROM MEDELLÍN CITY OF COLOMBIA
}

\begin{abstract}
:
The way of advertising has changed. Different audiences require companies to be closer to and pay attention to the problems that arise today, for this reason, Below The Line advertising, hereinafter BTL, through non-massive forms of communication generates marketing strategies for certain groups of the population with the objective of publicizing or generating a purchase intention towards a product. Which must occur within the parameters of sustainability. What is understood as the proper use of resources and the correct disposition of them to meet new objectives, that is, understand the use of resources as part of a chain that does not end with a single use within a single marketing action. These practices are part of Corporate Social Responsibility, hereinafter CSR, which is the active contribution that companies make to society, the economy and the environment where they carry out their activities. Therefore, in the case of the BTL Marketing industry of the city of Medellín, it will be identified if the practices described above are applied, in order to generate strategies applicable to the subsector object of this study.
\end{abstract}

Keywords: Sustainability, Social responsibility, advertising Below the line 


\section{INTRODUCCIÓN}

Según la unidad de política ambiental del DNP desde la década de los 90, el planeta se encuentra en una crisis ambiental por: 1) la explotación desmedida de los recursos naturales, generando escasez 0 incluso el riesgo de extinción de diversas especies, 2) la emisión de gases como el dióxido de carbono, que tienen efectos en el calentamiento de la tierra y los cambios de clima, 3) la producción de sustancias que destruyen la capa de ozono, que tienen como efecto la proliferación de rayos UV, con grandes consecuencias para la salud humana. Entre los países más contaminantes o que más impacto tienen en el sobrecalentamiento de la tierra, la perdida de la capa de ozono y la disminución de recursos, están los países industrializados que sus acciones tienen un impacto a nivel global. (1995)

La economía lineal es entendida como aquella que consiste en tomar los recursos para producir y después desecharlos, no hay un ciclo de reutilización, lo que lleva a más producción y, por lo tanto, más ventas. Por esto mismo, este tipo de economía en la actualidad se ha criticado y se ha buscado una manera de llevar un modelo sostenible del mercado.

La cadena lineal de extracción - producción - desecho, la cual agota los recursos naturales y genera residuos, está profundamente defectuosa y puede ser reemplazada por un modelo restaurativo en el que los residuos no existan como tales, sino que estos sean alimento para lo siguientes ciclos. (MacArthur, 2012, citado en Scheel y Aguiñaga, 2017).

La necesidad de cuidar los recursos e igualmente de seguir produciendo ha generado nuevos modelos económicos con impacto social y ambiental positivo como es la economía circular, que no permite el desecho o fin de un producto sino lleva a la reutilización de este.

Para Ellen MacArthur (2013) la economía circular: es aquella que es restaurativa y regenerativa a propósito, y que trata de que los productos, componentes y materias mantengan su utilidad y valor máximos en todo momento, distinguiendo entre ciclos técnicos y biológicos. Se concibe como un ciclo de desarrollo positivo continuo que preserva y mejora el capital natural, optimiza los rendimientos de los recursos y minimiza los riesgos del sistema al gestionar reservas finitas y flujos renovables. (p. 5).

De acuerdo a la oficina del presidente de La Asamblea general de Las Naciones Unidas La sostenibilidad o desarrollo sostenible se define como la satisfacción de las necesidades actuales sin comprometer los recursos de las generaciones futuras como se manifiesta en el informe titulado "Nuestro futuro común" para la Comisión Mundial sobre el Medio Ambiente y el Desarrollo (1987), donde también se afirma que el desarrollo sostenible consta de tres pilares principales que son el desarrollo económico, el desarrollo social y la protección del medio ambiente. 
Todo país que hace parte de las Naciones Unidas, asi como su industria, debe planear sus agendas, proyectos y planes hacia el desarrollo de sus actividades de una manera consciente. Por lo tanto, el conocimiento del impacto que tengan las empresas con la sociedad, la economía y el medio ambiente en su sitio de influencia debe ser un recurso de alta relevancia en la planeación y el desarrollo de su actividad económica.

De esta manera las empresas que hacen publicidad BTL deben dentro de sus objetivos de desarrollo tener en cuenta 1) el impacto que van a generar a la sociedad donde prestan sus servicios, 2) el impacto global que se derivara del uso de los recursos con la realización de su objeto social, que en este caso es el mercadeo no tracional a traves de medios no masivos. Dado que en Colombia este tipo de agencias son un segmento de la economía ha venido creciendo a un ritmo en promedio del $6 \%$ anual, y que la inversión en este tipo de publicidad según reporta la central de medios Asomedios para la fecha de publicación del artículo "BTL el nuevo protagonista de la publicidad" del diario La República (2014) sobrepasó la inversión en medios tradicionales como radio y televisión entre otros.

Por lo tanto, -específicamente en la ciudad de Medellín, potencial eje de la economía naranja en el país, según lo anunció El Presidente Iván Duque (2019) en el encuentro "Medellín Ciudad Creativa"- se busca identificar el conocimiento que tienen las empresas de publicidad BTL sobre prácticas sostenibles en el desarrollo de su actividad. Para, de esta manera llegar a un análisis cuantitativo y proponer estrategias que contribuyan a prácticas sociales, medio ambientales y economicamente responsables. Por lo tanto, se presenta la siguiente pregunta de investigación ¿Cúal es el conocimiento sobre las prácticas sostenibles por parte de los gerentes de las empresas de publicidad no tradicional en Medellín?. Los objetivos de investigación son los que se presentan a continuación, medir el conocimiento de los empresarios de las agencias BTL de la ciudad de Medellín sobre las prácticas sostenibles utilizadas en sus empresas, a partir de una investigación a través de contextualizar al lector sobre la agenda mundial de sostenibilidad, obtener información a través de datos primarios y secundarios de empresas BTL que prestan sus servicios en la ciudad de Medellín y proponer oportunidades de mejora en las practicas actuales de las agencias BTL.

\section{METODOLOGíA}

La investigación por realizarse será descriptiva para observar y describir el comportamiento de las empresas respecto al desarrollo de sus actividades de una manera sostenible, con el fin de reconocer las prácticas realizadas en la ciudad de Medellín y así mismo, generar estrategias que ayuden a las empresas a tener un impacto positivo a la sociedad. El trabajo mostrará estudios mediante métodos deductivos e inductivos. 
De manera deductiva se presentará el análisis y comprensión de 6 tratados universales sobre sostenibilidad como base de estudio básico, además de la investigación de textos o tesis sobre este mismo tema y el sector de publicidad BTL, como método de reconocimiento del entorno para entender cómo las empresas en determinado lugar, en este caso Medellín, entienden y trabajan hacia el desarrollo sostenible. Por otro lado, de manera inductiva se hará una investigación cuantitativa mediante encuestas y entrevistas que se emplearán como instrumento de recolección de datos sobre el conocimiento teórico del desarrollo sostenible que tienen los gerentes de las empresas y así mismo el empleo de este en sus prácticas laborales. Estas entrevistas debido a los lineamientos de distanciamiento social serán realizadas vía virtual con la herramienta -Forms de Google a 30 empresas que prestan sus servicios en la ciudad, como estudio de comportamiento y reconocimiento de sus labores. En adición, con la información recolectada, se busca identificar oportunidades de mejora que ayuden a las empresas del sector BTL a implementar la sostenibilidad como parte de sus objetivos corporativos.

Para entender la muestra a estudiar se tomará como fuente de información La edición el top de las 300 empresas de la comunicación de la revista P y M (2019). Listado en el que se encuentra el subsector de promo y activacion- BTL con 132 empresas que lideran en ventas y estrategias, de las cuales en Medellín figuran solamente 6 con su casa matriz en la ciudad. No obstante, lo mecionado anteriormente no afirma que estas sean las únicas agencias funcionando en la ciudad, ya que están también las que tienen casa matriz en Bogotá o Cali, que prestan sus servicios a anunciantes de la ciudad -126 restantes del listado de la revista especializada. El Universo según los datos obtenidos de la fuente es de 132 empresas en total para promo y activacion en el país, siendo la muestra por tomar de 32 empresas un 22,7\% del sector, un porcentaje significativo para obtener datos acertados. La revista $\mathrm{P}$ y $\mathrm{M}$ es la única especializada en el país sobre publicidad y tiene circulación trimestral. Las empresas que aparecen en este listado son elegidas por ingresos operacionales, según información reportada por cámaras de comercio y supersociedades. La numero 1 reporta 183.000 millones en ventas y la numero 132 tiene 1.000 millones a corte del 31 de diciembre de 2018.

\section{Revisión de Literatura}

El desarrollo sostenible es la manera de ejercer en los procesos, en este caso, de la economía o producción, un equilibrio entre los recursos y quienes los necesitan sin comprometer al medio ambiente a un impacto negativo del cual no se pueda recuperar, a través del uso adecuado, generando métodos de reutilización para continuar satisfaciendo las necesidades económicas, sociales y ambientales.

Teniendo en cuenta que las pequeñas y medianas empresas (MIPYMES) son en su mayoría el tejido empresarial de Colombia generando el 28\% del PIB y ofreciendo alrededor del 67\% de empleo (Dane, 2017, citado en Dinero, 2017) se entiende que 
es un público objetivo adecuado para poder hablar de la economía en Colombia y así mismo, identificar -dependiendo del sector- su responsabilidad en lo económico, ambiental y social.

Como resultado de la investigación sobre la responsabilidad de las empresas se entiende que el modelo económico actual debe cambiar a uno más sostenible:

La economía consumista medra con el movimiento de bienes, y cuanto más dinero cambia de mano tanto más florece. Y cada vez que hay dinero que cambia de mano hay producto de consumo que van a parar a la basura. Por lo tanto, en una sociedad de consumidores la búsqueda de la felicidad - el propósito invocado con más frecuencia y utilizado como carnada en las campañas publicitarias destinadas a atizar el deseo de los consumidores de desprenderse de su dinero (dinero ganado o dinero que uno espera ganar) - pasa de estar enfocada en producir cosas 0 apropiárselas (ni hablar de almacenarlas) para enfocarse en su eliminación. (Bauman, 2007)

Por esto, el modelo de economía circular es el adecuado para hablar de sostenibilidad creando empresas responsables, entendiendo este como:

un modelo económico basado en el principio de «cerrar el ciclo de vida» de los recursos de forma que se produzcan los bienes y servicios necesarios al tiempo que se reduce el consumo y el desperdicio de energía, agua y materias primas. Este modelo de ciclos cerrados de producción y consumo conserva los recursos naturales y contribuye al desarrollo sostenible. (Universidad Verde, 2016)

Además, la responsabilidad social de las empresas no sólo es en el aspecto ambiental sino también en lo económico y social, pues esto permite un desarrollo acorde de las sociedades, teniendo en cuenta que "el desarrollo que satisface las necesidades del presente sin poner en peligro la capacidad de las futuras generaciones para satisfacer sus propias necesidades" (Naciones Unidas, 1987, citado en Llorente \& Cuenca, 2011).

Para que una empresa pueda sobrevivir, según Sánchez, 2012, a medio-largo plazo deben ser económicamente viables, medioambientalmente sostenibles y socialmente responsable con sus grupos de interés o stakeholders, término que refiere "sólo a aquellos individuos o grupos de los que la organización necesita para sobrevivir a considerar a cualquiera de ellos que tenga un interés legítimo en la organización o se vea afectado por ella". (Caballero, s.f.).

Por esto, las organizaciones gubernamentales han tomado posición y anunciado tratados o formas de manejar el desarrollo sostenible y lo que este conlleva. Así, con base en estos poder generar estrategias que apoyen esa normatividad dada. 
A continuación, se mostrarán los tratados más importantes.

\section{Tratados mundiales sobre sostenibilidad}

- $\quad$ Agenda 2030 para el desarrollo sostenible.

El Programa de las Naciones Unidas para el Desarrollo (PNUD) en 2015 creó los 17 Objetivos de Desarrollo Sostenible con el fin de equilibrar una sostenibilidad ambiental, económica y social. Cada objetivo tiene un fin específico dependiendo de la problemática a vencer. En este caso el que se trabajará, es el objetivo 11 "Ciudades y comunidades sostenibles".

Según el PNUD (2015) en el año 2050 la población urbana podría llegar a 6,5 mil millones de personas, representando el 80\% del PIB global, el 60 al $80 \%$ del consumo de energía y $70 \%$ de las emisiones de carbono, significando que los sectores urbanos deben volverse lugares sostenibles. "No es posible lograr un desarrollo sostenible sin transformar radicalmente la forma en que construimos y administramos los espacios urbanos", además de ir mejorando "la planificación y gestión urbana de manera que sea participativa e inclusiva".

\section{- $\quad$ Agenda 21 de la Declaración de Rio Sobre el Medio Ambiente y el Desarrollo}

Esta agenda, propuesta por la Organización de las Naciones Unidas (ONU) en 1992, propone que los gobiernos locales de cada país ejecuten sus propios procesos para generar un lugar sostenible con el medio ambiente, evitando perjudicar al fomentar prácticas o estrategias que permitan preservar los ecosistemas, mediante aspectos como la innovación.

La Asociación Española para la Calidad, citando a la Organización de las Naciones Unidas, presenta los temas dado en la agenda organizada en cuatro sesiones:

- Sección I. Dimensiones sociales y económicas: la agenda propone una cooperación internacional para las luchas como la pobreza, las modalidades de consumo, dinámicas de sostenibilidad, protección humana, fomento del desarrollo de los recursos humanos e integración del medio ambiente.

- Sección II. Conservación y gestión de los recursos para el desarrollo: se refiere a la protección de la atmósfera, planificación de los recursos, lucha contra la deforestación, prevención de los ecosistemas frágiles, desarrollo rural sostenible, conservación de la diversidad biológica, protección de los océanos y de los recursos del agua, gestión racional de los desechos peligrosos o tóxicos.

- Sección III. Fortalecimiento del papel de los grupos principales: la mujer, la infancia, juventud, poblaciones indígenas, organizaciones no gubernamentales, empresas, trabajadores y sindicatos son grupos primarios para lograr un desarrollo sostenible. 
- Sección IV. Medios de ejecución: mecanismos de financiación, tecnología ecológica, ciencia, educación y capacitación, arreglos y mecanismos institucionales internacionales e información se toman como métodos para lograr un desarrollo sostenible.

- Protocolo de Kyoto El protocolo de Kyoto se rige en el cuidado del cambio climático en la prevención de las emisiones que producen una problemática en el aire, propone ocho objetivos para la reducción de los gases invernadero, dos de estos que funcionan en el sector económico de servicios son: (Naciones Unidad, 1998, p. 2)

- Fomento de la eficiencia energética en los sectores pertinentes de la economía nacional.

- Investigación, promoción, desarrollo y aumento del uso de formas nuevas y renovables de energía, de tecnologías de secuestro del dióxido de carbono y de tecnologías avanzadas y novedosas que sean ecológicamente racionales.

\section{- Convención Marco de las Naciones Unidas sobre el cambio climático}

Las Naciones Unidas (1992) presentó un tratado en el que los actores (de países desarrollados como en vía de desarrollo) deben velar por una sostenibilidad para las presentes y futuras generaciones preservando el derecho al desarrollo sostenible y su promoción. Tomando medidas para prever, prevenir o reducir las causas del cambio climático y así mismo disminuir las consecuencias, promocionando un sistema económico y vigilando un crecimiento económico. Formulando y aplicando programas nacionales 0 regionales con medidas orientadas a mitigar el cambio climático a través de tecnologías, prácticas y procesos que controlen para una adecuada aplicación y difusión, apoyando la investigación científica, tecnológica, técnica, socioeconómica y la educación, capacitación y sensibilización para estimular la participación de todos en los procesos de desarrollo.

\section{- Pacto Mundial}

El Pacto Mundial es una iniciativa dada por las Naciones Unidas (1999) para promover un comportamiento responsable de las empresas en cuatro grandes temas: crear normas laborales, velar en contra de la corrupción en las actividades, proteger el medio ambiente y velar por los derechos humanos. A este Pacto se encuentran adheridas 12.500 entidades de más de 160 países.

Respaldado por los CEOs de las empresas que lo componen, el Pacto Mundial es un marco práctico para desarrollar, implantar y divulgar políticas y prácticas de sostenibilidad empresarial, ofreciendo a sus signatories una amplia gama de recursos y herramientas de gestión para ayudarles a implementar modelos de negocio y desarrollo sostenible. (Pacto Mundial, s.f.). 


\section{- Libro verde}

Esta estratégia creada por la Comisión de las Comunidades Europeas tiene como principio generar una discusión sobre cómo fomentar un debate de responsabilidad social siguiendo líneas políticas para un desarrollo adecuado empresarial.

Propone fomentar prácticas correctas de las empresas; velar por la coherencia entre las políticas nacionales y los estándares internacionales; fomentar el partenariado entre empresa e interlocutores sociales; promover instrumentos de evaluación; animar a las empresas a adoptar un enfoque proactivo en relación al desarrollo sostenible; crear foros multistakeholders e incorporar prácticas de responsabilidad social en la gestión de gobierno. (Observatorio de Responsabilidad Social Corporativa, 2001).

\section{- $\quad$ Ley 99 de 1993}

Esta es la Ley General Ambiental de la república de Colombia que fundamenta la politica de medio ambiente en el pais según los principios universales de Desarrollo Sostible derivados de La Declaración de Rio de 1992 sobre medio ambiente y desarrollo.

Por la cual se crea El Ministerio del Medio Ambiente, se reordena el Sector Publico encargado de la gestión y conservación del medio ambiente y los recursos naturales renovable, se organiza el Sistema Nacional Ambiental SINA y si dictan otras disposiciones. (Ley General Ambiental de Colombia,1993)

Cada tratado o normativa creada por las diferentes organizaciones mundiales presenta una serie de lineamientos generales para los gobiernos, personas y empresas que tienen como deber adaptarlas al entorno en el que se encuentran para la generación de desarrollo sostenible y responsabilidad social, teniendo en cuenta que es una construcción en conjunto obligatoria.

Ahora bien, conociendo sobre estos tratados y La Ley ambiental para Colombia que exigen practicas sosteibles en las empresas, mediante la investigación a realizarse conoceremos si realmente las empresas cumplen esta normatividad 0 al menos conocen sobre el tema de la sostenibilidad y las diferentes teorías que la apoyan.

\section{Resultados}

Al enfocarse en la ciudad de Medellín, específicamente en las empresas en el sector de publicidad BTL, se evidencia que el conocimiento y la misma ejecución de sostenibilidad es media, donde algunas de las empresas no son todavía lo suficientemente sostenibles en los tres aspectos a tomar en el presente trabajo: económico, social y ambiental. 
El sector BTL es un sector nuevo en la economía, que está tomando auge poco a poco, puesto que las marcas están cambiando la forma en la que ven la publicidad y buscan nuevas maneras de llegar a los clientes, se están cambiando de los medios de comunicación convencionales a los no convencionales, por esto, el constante crecimiento del BTL que se ha visto en los últimos años y dado a que es un mercado innovador (de sus productos o servicios) ha llevado a que la sostenibilidad de aquellas empresas no sea una factor importante para las organizaciones.

En países donde se desarrolla mucho el BTL, existen agencias que operan más bien como consultoras, adaptando canales de comunicación y adaptándose en función de los presupuestos disponibles por el cliente. Por estas ventajas es que la tendencia a utilizar BTL aumenta. Por ejemplo, en Estados Unidos el 70\% del presupuesto delimitado para publicidad por parte de las empresas es orientado a este tipo de "acciones"; en Latinoamérica, durante la última década, se ha desarrollado mayormente en países como México y Argentina. (Garrido, 2010, p. 38).

Al ser un sector nuevo, su mayor problema es la desinformación o el poco conocimiento que tienen las nuevas empresas en este aspecto, debido a que desconocen las maneras en las que se pueden generar una empresa sostenible por el temor a cómo generar una economía renovable o los recursos que necesitan, ya sean económicos, tecnológicos o humanos.

Entre los resultados dados se identifica que las empresas BTL en Medellín apenas están iniciando una visión sostenible, puesto que en su ejecución estas se encuentran en un nivel básico, en una etapa primaria o no lo ejecutan del todo. Esto se evidencia en las dos preguntas iniciales de la entrevista, en la primera a la muestra elegida se le pregunto sobre su conocimiento de los Objetivos de Desarrollo Sostenible de las Naciones unidas y encontramos que un $56 \%$ de los lideres encuestados no los conocen. De igual manera, nuestra segunda pregunta se referia hacia el conocimiento sobre la Ley 99 de 1993, donde el desconocimiento fue aun mayor por 4 puntos, $60 \%$. 
Tabla I

Resultados encuesta, pregunta 2.

2. ¿Conoce los Objetivos de Desarrollo Sostenible de las Naciones Unidas?

Más detalles
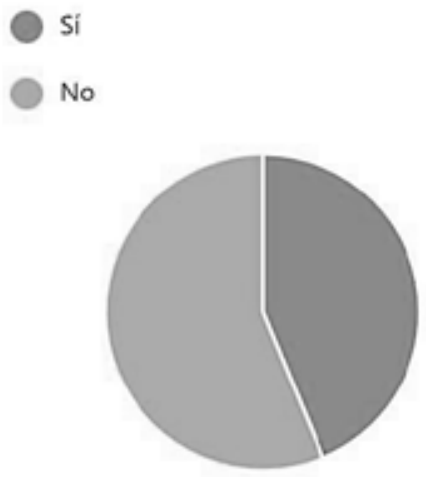

Tabla II

Resultados encuesta, pregunta 3.

3. ¿Está familiarizado con la Ley 99 de 1993 ? Más detalles
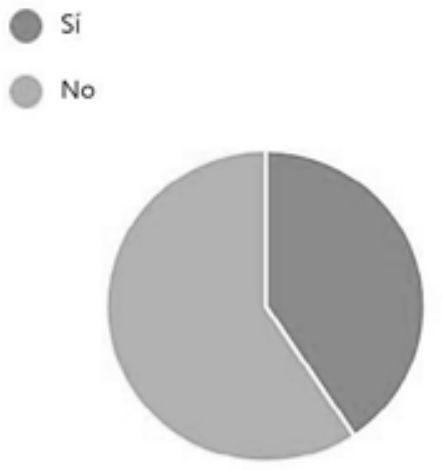

Según los pronósticos que se tenían sobre el desconocimiento por las empresas, en nuestro cuestionario se realizaron preguntas de conceptos que podrían ser más conocidos por los gerentes encuestados, como por ejemplo esta cuarta pregunta que sencillamente preguntaba sobre el cocnomiento del termino economía circular. Aun si, se encontró que un $37,5 \%$ no conocia el término que enmarca todo el conocimiento del desarrollo sostenible. 
Tabla III

Resultados encuesta, pregunta 4.

\section{4. ¿Sabe que es la economía circular?}

\section{Más detalles}
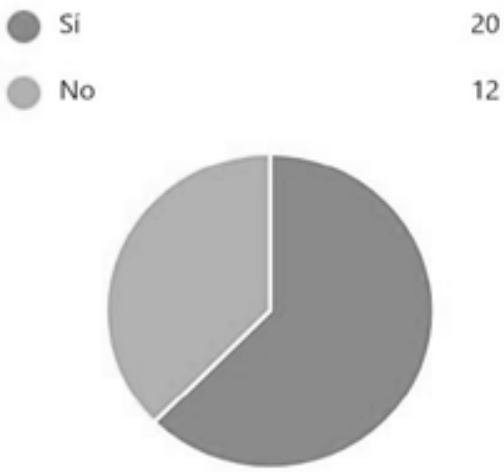

Nuestra siguiente pregunta era una autoevaluación a cada empresa sobre que tan sostenible era en sus prácticas. Efectivamente, siendo coherentes con las respuestas anteriores, su calificación fue media, dado al poco conocimiento por mas de la mitad de la muestra sobre las leyes, prácticas y tratados que enmarcan esta agenda.

Tabla IV

Resultados encuesta, pregunta 5.

5. En una calificación de 1 a 5 ¿qué tan sostenible es la empresa en la que usted presta los servicios?

\section{Más detalles}

\section{2}

Respuestas

\subsection{6}

\section{Promedio}

Uno de los tres aspectos fundamentales que propone la sostenibilidad es la sociedad, el entorno donde se ejerce la actividad economica y sus posibles afectados, por eso la investigación sobre el impacto de las practicas que realizan las empresas es clave al determinar si se está contribuyendo positivamente 0 al 
contrario afectando una comunidad. En las empresas encuestas, solamente un $25 \%$ de las mismas los apoyó proyectos de investigación hasta el momento de la entrevisa, lo cual nos demuestra que partiendo desde el desconocimiento de las normas, practicas y tratados se derivan las cifras bajas de practicas sostenibles en La Ciudad.

\section{Tabla V}

6. En la empresa que usted presta sus servicios ¿apoya proyectos de investigación a favor de la sostenibilidad en la comunidad?

Más detalles
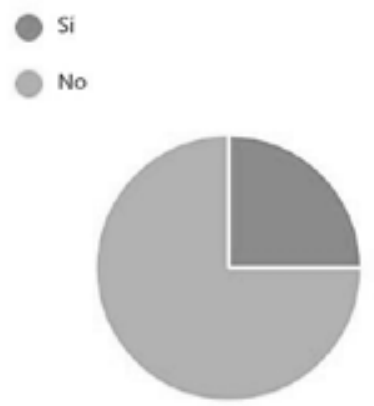

Así, el poco desarrollo sostenible que se realizan las agencias BTL de Medellin como fue mencionado anteriormente, se debe a la desinformación que hay del tema y a cómo se debe realizar. Las empresas no conocen las agendas mundiales que las rigen, ni mucho menos La Ley Colombiana que lo regula, por el contrario, en la siguiente grafica se observan los resultados sobre lo que piensan los empresarios que son los principales retos para ser una industria sostenible.

Tabla VI

Resultados encuesta, pregunta 11.

11. De las siguientes opciones, ¿cuál cree que es la barrera más grande al generar o ejecutar iniciativas sostenibles?

Mas detalles
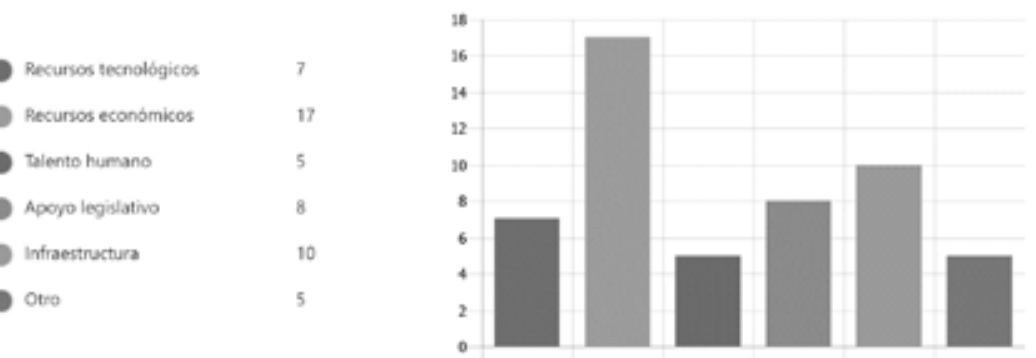
Como podemos notar en la gráfica, el mayor reto que indican los empresarios son los recursos economicos, seguido de la infraestuctura y los recursos tecnologicos. Lo cual realmente es un hallazgo interesante ya que podria demostrarse que realmente es el desconocimiento el causal de la baja sostenibilidad. Puesto que reportan en su mayoria no tener conocimiento sobre las agendas y normas existentes y aun asi el mayor reto para ellos es el recurso economico. Teoria que apoya la organización internacional del trabajo en el texto seguido a este parrafo.

Entre los principales obstáculos internos para las actividades y el crecimiento de las empresas pueden enumerarse la falta de competencias de gestión, una información y un conocimiento de los mercados y las finanzas limitados, la falta de sensibilización sobre el valor de los servicios de apoyo a las empresas, el uso de tecnologías obsoletas, 0 una falta de prácticas responsables en el lugar de trabajo que produce un bajo nivel de productividad. (Organización Internacional del Trabajo, p. 3, 2004)

Sin embargo, encontramos que las empresas encuestadas respondieron que si garantizaban el cuidado del medio ambiente en su mayoria, ademas de conocer los impactos derivados por la realización de sus actividades, lo cual no es coherente con la respuesta sobre la existencia de los sistemas de medicion ya que un $78 \%$ de las empresas no los tienen.

\section{Tabla VII}

Resultados encuesta, pregunta 7.

\section{7. ¿La empresa garantiza el cuidado del medio ambiente?}
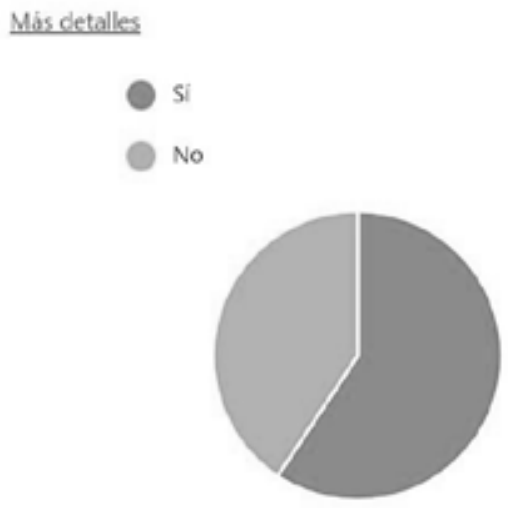
Tabla VIII: Resultados encuesta, pregunta 8.

Elaboración propia. Tomado de Forms.

8. ¿Conoce los impactos (positivos o negativos)

que puede tener la empresa en la sociedad

respecto a la sostenibilidad?

Mis detalles

No

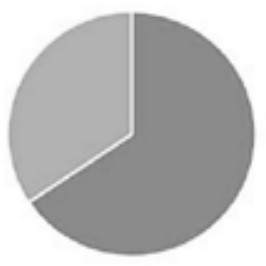

Tabla IX

Resultados encuesta, pregunta 9.

9. ¿La empresa emplea sistemas de medición para conocer el impacto ambiental?

Más detalles
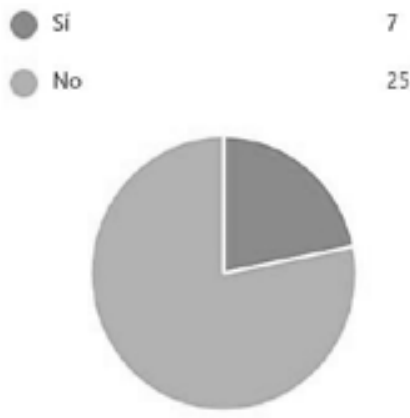

Los impactos y externalidades que una empresa tiene en el medio ambiente son en grandes cantidades, estas son las que más involucradas están en el uso de recursos, por lo tanto deben velar en el efecto que tienen, además que en el último siglo la intervención humana en el medio natural se ha elevado en niveles altos, es tanto que se ha dicho que "un uso más equitativo y racional de la tecnología y de los recursos naturales se concibe como parte de la solución a problemas endémicos como el hambre, la pobreza 0 determinadas enfermedades en el mundo" (Remacha, 2017). 
Las empresas difícilmente podrán definir su estrategia y desarrollar el negocio sin atender a los ODS, que recogen las preocupaciones de los grupos de interés a nivel mundial.

La consecución de los ODS creará el escenario idóneo para que el sector privado pueda desarrollar su actividad y eliminará obstaculos al crecimiento, frecuentes en sociedades injustas y desiguales. (Remacha, 2017).

\section{CONLUSIONES}

Teniendo en cuenta que una empresa sostenible no solo se refiere al impacto ambiental sino también al económico y social, según la OIT (Organización Internacional del trabajo) "en las empresas y en el mundo laboral, las esferas social, económica y ambiental del desarrollo sostenible interactúan de forma inseparable. Las empresas sostenibles alinean el crecimiento empresarial y la creación de empleos productivos y de trabajo decente con objetivos de desarrollo sostenible" (p.2, 2004). Las organizaciones a implementar un modelo de trabajo sostenible no sólo apoyan en el cuidado del medio ambiente, sino también pueden crecer al elevar su productividad, disminuir gastos y llamar la atención de la sociedad al apoyar el talento humano.

El problema que se evidencia del análisis de las empresas al no ser sostenibles es el poco interés que se le da a este aspecto, al no tomar la misma importancia con las labores de cada organización y la desinformación o el poco conocimiento que hay alrededor del tema. Las empresas desconocen las normas que rigen y obligan un trabajo sostenible, además del temor de una pequeña o media empresa que está empezando a invertir en sostenibilidad por los recursos económicos. Para generar un camino sostenible, como un objetivo laboral, se debe tener un direccionamiento estratégico puesto que "es un modelo de gestión que se caracteriza por tener una visión directiva global y una gestión adecuada de recursos, para proporcionar capacidades, fortalecer y facilitar el cumplimiento de los propósitos organizacionales". (Briceño, 2015)

Aunque hay un conocimiento por casi la mitad de la muestra investigada sobre los tratados generales para ser una empresa sostenible, es envidente que sigue estando el poco conocimiento respecto a este.

El 68.75\% de las empresas BTL en Medellín encuestadas no tienen un conocimiento amplio de lo que significa orientarse por un criterio de sostenibilidad, es un sector que apenas está iniciando y que por lo tanto tiene un camino largo a tomar para llegar a ser socialmente responsables, la desinformación sigue presente, por lo tanto es necesario educar al empresariado y que esto permita que las organizaciones puedan a mediano o a largo plazo convertirse en lo que la sociedad necesita, así velando por el interés común. 
Así mismo, se deben conocer los entes de control y los sistemas de medición de las empresas para determinar qué tan sostenibles son, además de permitir conocer si hay un correcto cumplimiento de la normatividad y de los tratados internacionales que rigen a las empresas a ser sostenibles, lo cual permitiría un mayor conocimiento para el direccionamiento correcto en el proceso de ser socialmente responsable.

Los impactos ambientales, sociales y económicos no se enfocan hacia un único grupo focal, sino que compete a todas las personas pertenecientes a una sociedad, el correcto manejo de los recursos se vuelve una necesidad de investigación para generar correctas estrategias que prevean colapsos en los sectores económicos, en las sociedades y en el medio ambiente.

\section{PROPUESTAS}

Se propone una agenda de educación en desarrollo sostenible para las empresas BTL de Medellín teniendo en cuenta los resultados de la investigación, teniendo como principal argumento que la sostenibilidad es sinónimo de innovación, confianza y reconocimiento de marca, además considerado las barreras que los gerentes de las Pymes plantean, como los recursos económicos seguido de la infraestructura. De esta manera se van a presentar y poner al alcance de los empresarios medidas y practicas exitosas en la escena mundial que se presentan a continuación:

Sistemas productos-servicios: dependiendo de los servicios tangibles 0 intangibles se pueden generar maneras que los clientes 0 usarios sigan consumiendo el producto/servicio a través de planes de fidelización mediante un resultado sostenible:

Existen diferentes tipos de sistemas productos-servicios: a) servicios orientados al producto, que simplemente añaden servicios, tales como acuerdos para mantenimiento o devoluciones, al sistema de producto existente; b) servicios orientados al uso, como arrendamiento, uso compartido o utilización en grupo; c) servicios orientados al resultado, que proporcionan resultados concretos, como por ejemplo la creación de un ambiente agradable en la oficina (Tukker y Tischner, 2006, citado en Cerdá, 2015).

Por ejemplo, crear planes renovo con productos de merchandising en los cuales después de un determinado tiempo estos puedan ser cambiados por otros, estableciendo alianzas con marcas.

Segunda vida de materiales y productos: reutilizar materiales para darle usos diferentes aprovechando el recurso varias veces. Esto se refiere, a cuando "una compañía puede recuperar y re acondicionar, de manera eficiente, sus productos 
después de su uso, y entonces poner los mismos productos en el mercado para obtener de esta manera un segundo o tercer ingreso" (Cerdá, p.13, 2015). Las empresas BTL pueden implementar la reutilización de stands e igualmente los elementos que se usan en estos.

Transformación de productos: Muchas veces un producto no se puede reutilizar en su totalidad, pero una parte de este sí, el aprovechamiento de estos recursos permite generar nuevos recursos a partir de uno, disminuyendo los gastos y aumentando las ganancias. Cuando una empresa BTL genera materia prima estas después pueden usarse para hacer otros elementos, ejemplo de un stand hecho de madera se puede reutilizar para hacer un trofeo de madera.

Consumo colaborativo: se refiere a cuando una o dos más empresas se unen en la distribución de un producto o en el reciclaje de este:

Se distinguen tres sistemas de consumo colaborativo: 1) mercados de redistribución, cuando se toma un artículo usado que tenía dueño y se pasa de un lugar en el que no es necesario a otro lugar o a otra persona donde si lo es, pero sin que se tenga que poseer el producto, 2) estilo de vida colaborativo: intercambio de recursos como dinero, habilidades y tiempo, 3) servicio de producto: pagar por el beneficio del producto. (Cerdá, p.13, 2015).

Por ejemplo, crear programas de reventa de los productos usados, puesto que estos pueden ser útiles para otra persona mientras que en la empresa se vuelve basura, como un disfraz usado en una activación de marca.

Así, para estar siempre con la idea de una empresa sostenible lo ideal es generar los servicios y productos con un fin renovable y diseñado para la sostenibilidad, teniendo en mente el ciclo de vida que se le va a dar a este, generando innovación en conjunto con las personas (sostenible con el talento humano), reduciendo gastos (sostenible con los recursos económicos), y con impacto ambiental menor (sostenible con el medio ambiente). Se propone la creación de la agremiación "BTL sostenible" encargada de realizar investigaciones sobre el impacto generado por la industria, además de crear un repositorio de información con los datos suministrados por las empresas del sector, el análisis del ciclo de vida de materiales y productos, maneras de disminuir costos y reconocimiento del talento humano, para posteriormente consolidar una base de datos de aliados con prácticas sostenibles. Se presenta un ejemplo de funcionamiento de la propuesta de la agremiación BTL Sostenible: Una agencia BTL de la ciudad contrata a un proveedor para realizar unos trofeos de reconocimiento para la convención de su cliente más importante, su proveedor, una empresa de manufacturas que trabaja el metal y el vidrio como principal insumo, le propone un trofeo que en su elaboración consume cantidades alarmantes de agua y emite dióxido de carbono con sus hornos de fundición. En este caso, BTL Sostenible haría una intervención al encontrar un productor de trofeos en el eje cafetero, empresa que utilizaría maderas de especies como el "pino pátula" cultivadas con fines comerciales, para la elaboración de artículos decorativos y 
trofeos que dentro de sus procesos el gasto de agua es casi mínimo y no funden ningún material, sino que es tallado a mano por artesanos; de esta manera se están creando productos de larga durabilidad a partir de una materia prima que convencionalmente es usada en la fabricación de elementos de un solo uso como el papel o cartón y así mismo generando empleo a familias de artesanos en una región donde los sectores de manufactura no tienen una producción masificada debido a la falta de tecnología y maquinaria especializada como podría haber en una ciudad principal. BTL Sostenible sería el primer cluster de educación en sostenibilidad de la ciudad enfocado hacia la industria, conociendo sus necesidades e integrando en un solo lugar: información sobre el ciclo de vida de productos comúnmente comercializados en la industria, productores con procesos sostenibles, agencias en la ciudad participantes activos, e incluso clientes que estén buscando agencias que cumplan con estos parámetros.

\section{REFERENCIAS}

ARTICULO DIARIO LA REPUBLICA "BTL el nuevo protagonista de la publicidad “ (2014). Sitio web: https://www.larepublica.co/consumo/btl-el-nuevoprotagonista-de-la-publicidad-2105744

ASAMBLEA GENERAL DE NACIONES UNIDAS, presidente del 65 periodo de sesiones sobre el Desarrollo sostenible. Sitio web: https://www.un.org/es/ ga/president/65/issues/sustdev.shtml

BRICEÑO, M \& MARTINEZ, E. (2015). Direccionamiento Estratégico. EAE. Sitio web: https://www.iberlibro.com/Direccionamiento-estratégico-MarthaCecilia-Briceño-Zamudio/20182852914/bd

CABALLER0, G. (s.f.). El poder de los "grupos de interés": un cambio en la actitud de la organización. Sitio web: https://www.fundacionseres. org/Lists/Informes/Attachments/1061/El\%20poder\%20de\%20los\%20 grupos\%20de\%20interés.pdf

CERDÁ, E. (2015). Economía circular. Proyecto EC02015-70349-P. Sitio web: https://dialnet.unirioja.es/servlet/articulo?codigo $=5771932$

COMISIÓN EUROPEA. (2001). Libro Verde: Fomentar un marco europeo para la responsabilidad social de las empresas. Observatorio de responsabilidad social corporativa. Sitio web: https://observatoriorsc.org/libro-verdefomentar-un-marco-europeo-para-la-responsabilidad-social-de-lasempresas/

ELLEN MACARTHUR FOUNDATION. (2013)."Hacia una economía circular. Motivos económicos para una transición acelerada". Sitio web: https:// www.ellenmacarthurfoundation.org/assets/downloads/publications/ Executive_summary_SP.pdf 
EXCLUSIVAS IMANARA. (2019). Fundamentos de la empresa responsable y sostenible. Sitio web: https://twitter.com/Imanara_ETT/ status/1156490954410532866

HARRISON, J. (junio, 2010). "El BTL, una alternativa publicitaria". Universidad Dr. José Matías Delgado. Sitio web: https://webquery.ujmd.edu.sv/siab/ bvirtual/BIBLIOTECA\%20VIRTUAL/TESIS/03/DGR/ADHB0001184.pdf

LEY GENERAL AMBIENTAL DE COLOMBIA -Diario Oficial No. 41.146, de 22 de diciembre. (1993). Organización de Estados Americanos. Sitio web: https:// www.oas.org/dsd/fida/laws/legislation/colombia/colombia_99-93.pdf

LLORENTE \& CUENCA. (2011). Sostenibilidad y Responsabilidad Social en la empresa. Sitio web: https://www.google.com/url?sa=t\&sour $c e=$ web\&rct $=j \& u r l=$ https://ideas.. lorenteycuenca.com/download/ Informes/110922_d\%2BiLL\%26C_CIES_Informe_Especial_RSE.pdf\&ved $=$ 2ahUKEwjlqp7j57TpAhWMTd8KHS12AvcQFjACegQIBBAB\&usg $=$ AOvV aw0Arhb8bQUmjxtH3sGOW-Ot

NACIONES UNIDAS. (1992). Convención Marco de las Naciones Unidas sobre el cambio climático. Sitio web: https://unfccc.int/resource/docs/convkp/ convsp.pdf

NACIONES UNIDAS. (1998). Protocolo de KYOTO de la convención Marco de las Naciones Unidas sobre el cambio climático. Sitio web: https://unfccc. int/resource/docs/convkp/kpspan.pdf

ORGANIZACIÓN DE LAS NACIONES UNIDAS. (s.f.). Agenda 21. Asociación Española para la Calidad. Sitio web: https://www.aec.es/web/guest/ centro-conocimiento/agenda-21

ORGANIZACIÓN INTERNACIONAL DEL TRABAJO. (2014). Empresas Sostenibles: creación de más y mejores empleos. Organización Internacional del Trabajo. Sitio web: https://www.google.com/url?sa=t \&source =web\&rct=j\&url=https://www.ilo.org/wcmsp5/groups/public/--ed_emp/---ed_emp_msu/documents/publication/wcms_185282.pdf\& ved=2ahUKEwjQmvGu4rPpAhWpTd8KHcWBBbIQFjABegQIAXAB\&usg $=$ AOvVaw3sMRJWcle4LSy6HaOwP7S7

Pacto Mundial . (s.f.). Diez Principios. Pacto Mundial. Sitio web: https://www. pactomundial.org/category/aprendizaje/10-principios/

Pacto Mundial . (s.f.). ¿Quienes somos?. Pacto Mundial. Sitio web: https:// www.pactomundial.org/category/aprendizaje/10-principios/

Planeación y desarrollo vol XXVI, No. 3, julio - septiembre 1995 Unidad de politica ambiental del DNP. Sitio web: https://colaboracion.dnp.gov.co/ CDT/RevistaPD/1995/pd_vXXVI_n3_1995_art.4.pdf 
PROGRAMA DE LAS NACIONES UNIDAS PARA EL DESARROLLO. (2015). Objetivo 11: Ciudades y comunidades sostenibles. Naciones Unidas. Sitio web: https://www.undp.org/content/undp/es/home/sustainabledevelopment-goals/goal-11-sustainable-cities-and-communities.html

REMACHA, M. (2017). Medioambiente: desafíos y oportunidades para las empresas. Sitio web: https://media.iese.edu/upload/ST0431.pdf

REMACHA, M. (2017). Empresa y objetivos de desarrollo sostenible. Sitio web: https://media.iese.edu/upload/ST0438.pdf

REVISTA DINERO. (2017). MIPYMES generan alrededor del $67 \%$ del empleo en Colombia. Sitio web: https://www.dinero.com/edicion-impresa/ pymes/articulo/evolucion-y-situacion-actual-de-las-mipymes-encolombia/222395

SÁNCHEZ, N. (2012). La sostenibilidad en el sector empresarial. Sitio web: https://upcommons.upc.edu/bitstream/handle/2099.1/18820/TFM NSanchez_La\%20sostenibilidad\%20en\%20el\%20sector\%20empresarial. pdf

SCHEEL, C \& AGUIÑAGA, E. (Septiembre, 2017) "Economía circular, una alternativa a los límites de crecimiento lineal". Sitio web: https://www. researchgate.net/publication/319839814_Economia_circular_una_ alternativa_a_los_limites_del_crecimiento_lineal

UNIVERSIDAD VERDE. (2016). La economía circular: qué es y cómo se aplica a la política local. Sitio web: https://www.google.com/url?sa=t\&source $=$ w eb\&rct=j\&url=http://universidadverde.es/wp-content/uploads/2016/08/ Que-es-la-economia-circular.pdf\&ved = 2ahUKEwjczPjf5LTpAhXPJt8KHW D2DPUQFjABegQIBRAB\&usg = AOvVaw0h2u45nHnuBHw-umdrvfxa

ZYGMUNT BAUMAN (2007). Vida de consumo. Sitio web: https:// estudioscultura.files.wordpress.com/2015/08/bauman_vida_de_ consumo_modo_de_compatibilidad.pdf 